\title{
PepN is the major aminopeptidase in Escherichia coli: insights on substrate specificity and role during sodium-salicylate-induced stress \\ Correspondence \\ Dipankar Nandi \\ nandi@biochem.iisc.ernet.in \\ Received 27 May 2003 \\ Revised 29 July 2003 \\ Accepted 13 August 2003

\author{
Dilip Chandu and Dipankar Nandi
} \\ Department of Biochemistry, Indian Institute of Science, Bangalore 560012, India

\begin{abstract}
PepN and its homologues are involved in the ATP-independent steps (downstream processing) during cytosolic protein degradation. To obtain insights into the contribution of PepN to the peptidase activity in Escherichia coli, the hydrolysis of a selection of endopeptidase and exopeptidase substrates was studied in extracts of wild-type strains and two pepN mutants, 9218 and $\mathrm{DH} 5 \alpha \Delta$ pepN. Hydrolysis of three of the seven endopeptidase substrates tested was reduced in both pep $N$ mutants. Similar studies revealed that hydrolysis of 10 of 14 exopeptidase substrates studied was greatly reduced in both $\operatorname{pep} N$ mutants. This decreased ability to cleave these substrates is $p e p N$-specific as there is no reduction in the ability to hydrolyse exopeptidase substrates in $E$. coli mutants lacking other peptidases, pepA, pepB or pepE. PepN overexpression complemented the hydrolysis of the affected exopeptidase substrates. These results suggest that $\mathrm{PepN}$ is responsible for the majority of aminopeptidase activity in E. coli. Further in vitro studies with purified PepN revealed a preference to cleave basic and small amino acids as aminopeptidase substrates. Kinetic characterization revealed the aminopeptidase cleavage preference of E. coli PepN to be Arg $>$ Ala $>$ Lys $>$ Gly. Finally, it was shown that PepN is a negative regulator of the sodium-salicylate-induced stress in E. coli, demonstrating a physiological role for this aminoendopeptidase under some stress conditions.
\end{abstract}

\section{INTRODUCTION}

The mechanisms involved in cytosolic protein degradation are central to regulating various aspects of cell biology, including cell cycle, disease progression, transcriptional regulation, antigen processing, stress responses, etc. Proteins targeted for degradation are unfolded and cleaved in an ATP-dependent manner to release large peptides, ranging from 3 to 25 amino acids (Maurizi, 1987; Coux et al., 1996; Gottesman, 1996; Kisselev et al., 1999; Zwickl et al., 2000). These are further trimmed, cleaved and/or degraded by several endopeptidases, tri- and di-peptidyl peptidases (Tamura, T. et al., 1996; Fukasawa et al., 1998; Osmulski \& Gaczynska, 1998; Tamura, N. et al., 1998; Geier et al., 1999; Wang et al., 2000), aminopeptidases and carboxypeptidases (Conlin \& Miller, 1995; Gonzales \& RobertBaudouy, 1996; Chandu \& Nandi, 2002; Franzetti et al., 2002) in an ATP-independent manner, also known as downstream processing. This general scheme of cytosolic protein degradation is conserved in all organisms, although the enzymes involved are distinct in different organisms. Most of the enzymes and their homologues that are

Abbreviations: AMC, 7-amino-4-methylcoumarin; $\beta \mathrm{NA}, \beta$-naphthylamide; Boc, butoxycarbonyl; Cbz, benzoyloxycarbonyl; NaSal, sodium salicylate; $p N A, p$-nitroanilide. upstream in the proteolysis pyramid, i.e. endopeptidases (e.g. Tricorn, tripeptidyl peptidase II, bleomycin hydrolase, thimet oligopeptidase), are present in selected organisms. However, the enzymes and their homologues involved in the latter steps of downstream processing, for example, leucine aminopeptidase and puromycin-sensitive aminopeptidase, are present in most organisms (Chandu \& Nandi, 2002). Also, prokaryotes display greater redundancy than eukaryotes in the key enzymes involved in protein degradation. For example, $20 \mathrm{~S}$ proteasomes, which are essential in eukaryotes (Coux et al., 1996), are not essential in Mycobacterium smegmatis (Knipfer \& Shrader, 1997). However, they are important only during heat shock in Thermoplasma acidophilum (Ruepp et al., 1998). Similarly, Lon and Clp, the key ATP-dependent proteases in Escherichia coli, are not essential but are required during some stress conditions (Gottesman, 1996; Kuroda et al., 2001).

Recently, characterizing enzymes involved in the ATPindependent process and studying their functional role has gained importance. Many of the studies have been done on archaeal and eukaryotic organisms. Enzymes involved in downstream processing, for example, tripeptidyl peptidase II, thimet oligopeptidase, bleomycin hydrolase, leucine aminopeptidase and puromycin-sensitive aminopeptidase, 
are also important in the trimming and degradation of major histocompatibility complex (MHC) class I binding peptides in mammals (Beninga et al., 1998; Stoltze et al., 2000; Saric et al., 2001; York et al., 2003). In T. acidophilum, this proteolytic process has been reconstituted in vitro (Tamura, N. et al., 1998) and crystal structures have been determined for most of the key enzymes (Lowe et al., 1995; Brandstetter et al., 2001; Goettig et al., 2002). Peptides released by $20 \mathrm{~S}$ proteasomes are further degraded by Tricorn endoprotease to short peptides which are in turn broken down into amino acids by the Tricorn interacting exopeptidases, F1, F2 and F3. In fact, a model of the Tricorn-F1 complex suggests that the $\beta 7$ propeller of Tricorn is used for substrate entry whereas the $\beta 6$ propeller is for product egress and may act as a docking site for binding the Tricorn interacting factor F1 (Goettig et al., 2002). Methionine aminopeptidases are essential in E. coli and Saccharomyces cerevisiae, demonstrating that cleavage of the N-terminal methionine from some proteins is critical for cellular function (Chang et al., 1989; Bradshaw et al., 1998). Peptidases in Lactococcus lactis are required for proteolysis and growth in milk (Mierau et al., 1996). The turnover of cellular proteins is reduced in multiple-peptidase mutants, but not in singlepeptidase mutants, in Salmonella typhimurium (Yen et al., 1980) and E. coli (Miller \& Schwartz, 1978; Conlin \& Miller, 1995). Due to the redundancy of peptidases in prokaryotes, there is little information available on the physiological roles of these enzymes.

PepN, also known to be the sole alanine aminopeptidase in E. coli, was identified 28 years ago (Lazdunski et al., 1975a, b). Recently, we initiated studies on enzymes involved in downstream processing in eubacteria. In the course of our studies, we identified PepN from E. coli to be responsible for cleaving Suc-LLVY-7-amido-4-methylcoumarin (AMC), a substrate cleaved by the 20S proteasome from all sources. PepN was further characterized with respect to its aminoendopeptidase activities (Chandu et al., 2003). PepN and its homologues are well conserved in all kingdoms and play a role in downstream processing during cytosolic protein degradation. We wished to study, using synthetic peptidase substrates, the substrate specificity of pure PepN. In addition, we wished to evaluate the contribution of PepN to overall cellular peptidase activities in extracts of wild-type and pepN mutant strains of E. coli. Previous studies had failed to demonstrate a phenotype for E. coli strains lacking pepN (McCaman et al., 1982; Bally et al., 1983). Therefore, we wished to investigate the role of PepN during different stress conditions. In this study, we demonstrate that PepN is responsible for the majority of the aminopeptidase activity in E. coli and that it prefers to cleave basic and small amino acids at the amino terminus of substrates. Furthermore, we show that PepN plays a negative role during sodium salicylate (NaSal)-induced stress.

\section{METHODS}

Strains, plasmids and overexpression of PepN. All E. coli strains used in the study were maintained in Luria-Bertani (LB) medium in the absence or presence of appropriate antibiotics: ampicillin $\left(100 \mu \mathrm{g} \mathrm{ml}^{-1}\right)$, tetracycline $\left(30 \mu \mathrm{g} \mathrm{ml}^{-1}\right)$ and kanamycin $\left(30 \mu \mathrm{g} \mathrm{ml}^{-1}\right)$. E. coli 9218 is an $N$-methyl- $N^{\prime}$-nitro- $N$-nitrosoguanidine-derived pepN mutant (Bally et al., 1983) and E. coli DH5 $\alpha \Delta p e p N$, a targeted deletion mutant of pepN, has been described previously (Chandu et al., 2003). pepN was overexpressed from pBM15, a pBR322-based vector harbouring pepN under the control of its endogenous promoter (Bally et al., 1984). In another construct, the E. coli K-12 pepN open reading frame was PCR-amplified and cloned into pBAD24, an $\mathrm{L}$-arabinose-inducible vector, and referred to as pBAD/K12pepN (Chandu et al., 2003). pepN was PCR-amplified from E. coli 9218 using gene-specific primers (Chandu et al., 2003) and cloned under the control of the L-arabinose-inducible promoter of pBAD24; this construct is referred to as $\mathrm{pBAD} / 9218 p e p N$ in the text. Strain DH5 $\alpha \Delta p e p N(\mathrm{pBAD} 24, \mathrm{pBAD} / \mathrm{K} 12 p e p N$ or $\mathrm{pBAD} / 9218 p e p N)$ was grown in the presence of $0 \cdot 4-2 \mathrm{mg} \mathrm{L}$-arabinose $\mathrm{ml}^{-1}$ for overexpression studies. The DNA sequence of K12pepN and 9218pepN was determined using M13 and pepN-specific primers. E. coli strains with Tn5 insertions in their peptidase genes ( $е$ epA, pepB and $p e p E)$, constructed in E. coli MG1655, were obtained from the Mutant strain collection, E. coli Genome Project, University of Wisconsin Madison, WI, USA (http://www.genome.wisc.edu/).

Generation of antiserum to PepN and Western analysis. Antiserum was raised against purified PepN by injecting $200 \mu \mathrm{g}$ of the pure protein per rabbit as an emulsion with complete Freund's adjuvant subcutaneously. Two booster injections of $100 \mu \mathrm{g}$ PepN per rabbit were given with incomplete Freund's adjuvant, after gaps of 2 weeks. One week after the second booster, the serum was obtained and stored at $-70{ }^{\circ} \mathrm{C}$ until further use. This antiserum against PepN specifically detects the enzyme from purified preparations as well as crude extracts, by both ELISA and Western blotting. However, pre-immune serum collected from the same rabbit before PepN immunization displayed negligible reactivity (data not shown). Western analysis was performed by separating the cellular extracts on a $10 \%$ SDS-PAGE gel and transferring them to a nitrocellulose membrane. After blocking overnight with $1 \%$ gelatin in $50 \mathrm{mM}$ PBS $\left(0.01 \mathrm{M} \mathrm{NaH}_{2} \mathrm{PO}_{4}, 0.04 \mathrm{M} \mathrm{Na}_{2} \mathrm{HPO}_{4}, 0.15 \mathrm{M} \mathrm{NaCl}\right.$, $\mathrm{pH} 7 \cdot 4)$, the pre-immune serum and $\mathrm{PepN}$-specific antiserum were used at a dilution of $1: 10000$. Goat anti-rabbit antibody conjugated to horseradish peroxidase (Bangalore Genei) was used as secondary antibody ( $1: 2000$ dilution). Antibodies bound to PepN were visualized by staining with hydrogen peroxide and 3,3'-diaminobenzidine (Sigma) in $50 \mathrm{mM}$ PBS. This antiserum specifically recognized PepN in cytosolic extracts from wild-type E. coli but not in cytosolic extracts from two strains lacking pepN, namely, 9218 and DH5 $\alpha \Delta$ pepN (Fig. 2a, b). Also, no cross-reactivity was found in cytosolic extracts from $M$. smegmatis or mouse liver with this antiserum (data not shown).

PepN purification, substrate hydrolysis assays and kinetic characterization. Overnight cultures of E. coli $\left(\mathrm{OD}_{600}\right.$ of $\left.\sim 2\right)$ were used as $0.5 \%(\mathrm{v} / \mathrm{v})$ inoculum and were grown for $\sim 10 \mathrm{~h}$ at $37^{\circ} \mathrm{C}$, washed, sonicated and centrifuged at $100000 \mathrm{~g}$ for $1 \mathrm{~h}$ at $4{ }^{\circ} \mathrm{C}$ to obtain cell-free extracts. For experiments using the pBAD24 system, cultures ( $1 \%$ inoculum) were grown for $4 \mathrm{~h}$ in the presence of appropriate amounts of L-arabinose. Assays with cellular extracts were performed using $25 \mu \mathrm{g}$ total protein, for both aminopeptidase and endopeptidase substrate hydrolysis. Results shown are representative of at least two independent extract preparations. PepN was purified from E. coli DH5 $\alpha$ transformed with pBM15 using conventional chromatographic procedures, as described previously (Chandu et al., 2003). Single-band purity was checked by separating PepN on SDS-PAGE and staining with Coomassie Brilliant blue R-250. Enzyme assays were performed as described previously (Chandu et al., 2003). Briefly, endopeptidase $(0.5 \mathrm{mM})$ and aminopeptidase $(1 \mathrm{mM})$ substrates were incubated with purified $\mathrm{PepN}$ in $20 \mathrm{mM}$ phosphate 
buffer, $\mathrm{pH} 8 \cdot 0$, at $37^{\circ} \mathrm{C}$. The endopeptidase substrates (Sigma) used in the study, with cleavage specificity in parentheses, are as follows: Boc-Leu-Arg-Arg-AMC (trypsin-like enzymes), Cbz-Ala-Arg-ArgAMC (trypsin-like enzymes), Cbz-Gly-Gly-Leu- $\beta$-naphthylamide $(\beta \mathrm{NA})$ (ClpYQ protease substrate), Cbz-Leu-Leu-Glu- $\beta \mathrm{NA}$ (postglutamyl peptidyl hydrolytic activity), Suc-Ala-Ala-Phe-AMC (chymotrypsin-like enzymes), Suc-Leu-Leu-Val-Tyr-AMC (chymotrypsin-like enzymes), Suc-Leu-Tyr-AMC (porcine calpain I- and II-like enzymes). The tripeptidyl and exopeptidase substrate used was Ala-Ala-Phe-AMC and the aminopeptidase substrates used were L-Ala- $p$-nitroanilide ( $p \mathrm{NA})$, L-Arg-AMC, L-Asp- $\beta \mathrm{NA}$, L-Asn- $\beta \mathrm{NA}$, L-Gly- $p$ NA, L-Leu- $p$ NA, L-Lys- $p$ NA, L-Met- $p$ NA, L-Phe- $p$ NA, L-Pro$\beta$ NA, L-Thr-AMC, L-Tyr-AMC and L-Val- $\beta$ NA. Purified PepN was used at $1 \mu \mathrm{g}$ for $2 \mathrm{~h}$ and $7 \cdot 5 \mathrm{ng}$ for $1 \mathrm{~h}$ for endopeptidase and aminopeptidase assays, respectively, and reactions were terminated with $100 \%$ ethanol. Free AMC or $\beta$ NA released after cleavage of peptide substrates was measured, using a spectrofluorimeter (Shimadzu), with excitation wavelengths of 370 and $335 \mathrm{~nm}$ and emission wavelengths of 430 and $410 \mathrm{~nm}$ for AMC-based and $\beta$ NA-based substrates, respectively. Similar assays with chromogenic substrates were performed and free $p \mathrm{NA}$ released was measured by studying the absorbance at $410 \mathrm{~nm}$ in the spectrophotometer (Shimadzu). The net increase in AMC or $p \mathrm{NA}$ released was calculated after subtraction with appropriate controls. Standard curves were plotted using known amounts of free AMC or pNA to calculate the amount released. Protein amounts were calculated using Bradford's reagent and known amounts of BSA (Sigma) were used to generate a standard plot. Specific activity was calculated as nanomoles of AMC or $p \mathrm{NA}$ released per microgram of protein per hour at $37^{\circ} \mathrm{C}$. Kinetic characterization of a particular substrate was done by performing enzyme assays with different concentrations of the substrate. Kinetic constants were obtained from the direct linear plots (Eisenthal \& Cornish-Bowden, 1974).

NaSal-induced stress. Single colonies of different E. coli strains were grown in $3 \mathrm{ml} \mathrm{LB}$ medium overnight, with appropriate antibiotics. Cell-free extracts of the strains were prepared after sonication and assayed for hydrolysis of Suc-LLVY-AMC or L-Ala-pNA, to confirm the authenticity of the cultures, as a standard practice. Each tested culture was streaked on one quadrant of appropriate plates and incubated at $37^{\circ} \mathrm{C}$ in the absence or presence of increasing amounts of NaSal. In addition, a plate streaked with different strains was incubated at $42{ }^{\circ} \mathrm{C}$ to study the effect of growth at high temperature. After growth for $\sim 10 \mathrm{~h}$, the images of plates were obtained with the ALPHADIGIDOC documentation system (San Leandro, CA, USA).

\section{RESULTS}

\section{Hydrolysis of peptidase substrates by pure PepN}

First, the cleavage specificity of pure PepN was studied, using a panel of endopeptidase and aminopeptidase substrates. PepN hydrolysed three of the seven endopeptidase substrates tested (Fig. 1a). Interestingly, pure PepN hydrolysed eight out of the 13 aminopeptidase substrates tested (Fig. 1b), confirming that PepN is a broad-specificity aminopeptidase (McCaman \& Villarejo, 1982). However, pure PepN hydrolysed basic (arginine and lysine) and small (alanine and glycine) amino acid substrates better than the rest (Fig. 1b), suggesting a preference for hydrolysis of these aminopeptidase substrates.

To obtain further insights into the cleavage preference of (a)

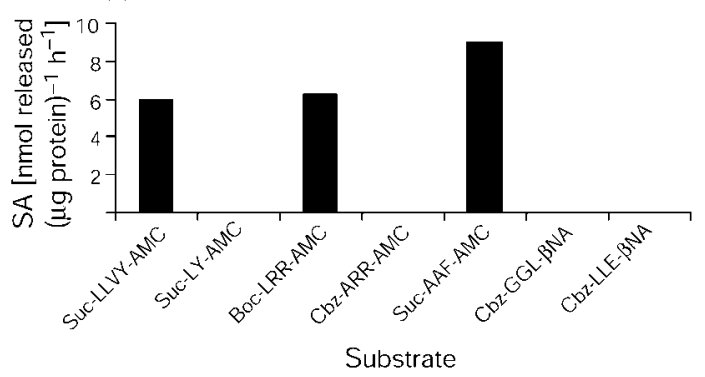

(b)

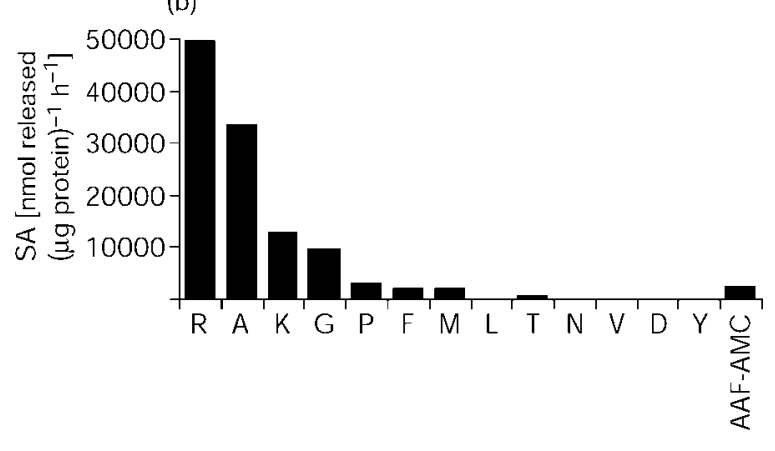

Fig. 1. PepN is a broad-specificity aminopeptidase. PepN was purified biochemically and assayed for its ability to hydrolyse a panel of endopeptidase (a) or exopeptidase (b) substrates. Pure PepN $(1 \mu \mathrm{g})$ was incubated with different endopeptidase substrates $(0.5 \mathrm{mM})$. To study the hydrolysis of exopeptidase substrates $(1 \mathrm{mM}), 7 \cdot 5 \mathrm{ng}$ of PepN was used. $x$-Axis labels in (b) represent standard single letter code for amino acids, which are present $\mathrm{N}$ terminus to the peptide bond hydrolysed. The free AMC, $\beta N A$ or $p N A$ released from these peptide substrates after hydrolysis by PepN was measured in a spectrofluorimeter (AMC and $\beta N A$ ) or spectrophotometer ( $p N A)$. SA, specific activity. Results shown are representative of at least two independent enzyme preparations.

PepN for synthetic substrates, kinetic characterization of selected peptidase substrates was performed (Table 1). Kinetic characterization revealed that PepN displays higher $k_{\text {cat }} / K_{\mathrm{m}}$ values for exopeptidase substrates than endopeptidase substrates. In our previous report (Chandu et al., 2003), we demonstrated that PepN is a better aminopeptidase than endopeptidase using L-Ala-pNA and Suc-LLVY-AMC. As these substrates were not similar, we selected the exopeptidase substrate AAF-AMC and its cognate endopeptidase substrate Suc-AAF-AMC for kinetic comparison. Although the $K_{\mathrm{m}}$ values of these substrates were comparable, PepN hydrolysed AAF-AMC with an $\sim 180$-fold higher $k_{\text {cat }} / K_{\mathrm{m}}$ value than Suc-AAF-AMC (Table 1). These results corroborate our previous observation that PepN is a better exopeptidase than endopeptidase. Significantly, the sole alanine aminopeptidase of E. coli, PepN, displayed an $\sim 8$ fold higher $k_{\text {cat }} / K_{\mathrm{m}}$ value for arginine compared to alanine at the $\mathrm{P} 1$ position (i.e. the amino acid present $\mathrm{N}$-terminal to the peptide bond that is hydrolysed by proteases/peptidases) of aminopeptidase substrates (Table 1). The preference of 
Table 1. Kinetic characterization of peptidase substrate hydrolysis by pure PepN

Cleavage of different peptide and amino acid substrates by pure PepN was performed with varying concentrations of substrates. Kinetic parameters were calculated from the direct linear plots. Data are representative of two to six independent enzyme preparations and are shown \pm SE.

\begin{tabular}{|lcccc|}
\hline \multirow{2}{*}{ Substrate } & \multicolumn{4}{c|}{ Kinetic parameter } \\
\cline { 2 - 5 } & $\boldsymbol{K}_{\mathbf{m}}(\boldsymbol{\mu} \mathbf{M})$ & $\left.\boldsymbol{V}_{\max }(\boldsymbol{\mu} \mathbf{M ~ s})^{-\mathbf{1}}\right)$ & $\boldsymbol{k}_{\text {cat }}\left(\mathbf{s}^{-\mathbf{1}}\right)$ & $\boldsymbol{k}_{\text {cat }} / \boldsymbol{K}_{\mathbf{m}}\left(\mathbf{s}^{-\mathbf{1}} \boldsymbol{\mu \mathbf { M } ^ { - \mathbf { 1 } } )}\right.$ \\
\hline L-Arg-AMC & $109 \pm 19 \cdot 0$ & $0 \cdot 069 \pm 0 \cdot 001$ & $946 \cdot 75$ & $8 \cdot 6800$ \\
L-Ala-pNA & $638 \cdot 6 \pm 13 \cdot 8$ & $0 \cdot 05 \pm 0 \cdot 0031$ & $692 \cdot 10$ & $1 \cdot 0830$ \\
L-Lys- $p$ NA & $533 \cdot 33 \pm 40 \cdot 8$ & $0 \cdot 013 \pm 0 \cdot 005$ & $181 \cdot 60$ & $0 \cdot 3400$ \\
L-Gly-pNA & $675 \pm 75 \cdot 03$ & $0 \cdot 006 \pm 0 \cdot 00002$ & $84 \cdot 70$ & $0 \cdot 1250$ \\
AAF-AMC & $559 \cdot 5 \pm 31 \cdot 5$ & $0 \cdot 5 \pm 0 \cdot 08$ & $51 \cdot 75$ & $0 \cdot 0924$ \\
Suc-LLVY-AMC & $40 \cdot 8 \pm 1 \cdot 25$ & $0 \cdot 0007 \pm 0 \cdot 00006$ & $0 \cdot 08$ & $0 \cdot 0019$ \\
Suc-AAF-AMC & $339 \pm 39$ & $0 \cdot 001 \pm 0 \cdot 0002$ & $0 \cdot 19$ & $0 \cdot 0005$ \\
\hline
\end{tabular}

PepN for arginine resulted from the high affinity for this substrate (low $K_{\mathrm{m}}$ ) compared to alanine. Kinetic parameters demonstrated a hierarchy of preference for hydrolysis of aminopeptidase substrates: Arg > Ala > Lys > Gly. Albeit the broad specific nature, kinetic characterization revealed that PepN displayed a preference for basic and small amino acids as $\mathrm{P} 1$ residues in aminopeptidase substrates.

\section{Characterization and mapping of the mutation in pepN from E. coli 9218}

In addition to displaying aminoendopeptidase activities (Chandu et al., 2003), our current results with pure PepN demonstrated that it has the ability to hydrolyse a wide variety of aminopeptidase substrates. This prompted us to check the contribution of PepN for overall peptidase activity in cell-free extracts of two pepN mutants, 9218 and DH $5 \alpha \Delta p e p N$, with their respective parent strains. We demonstrated the construction of strain E. coli DH $5 \alpha \Delta p e p N$, with a targeted deletion in pepN and lacking PepN activity, previously (Chandu et al., 2003). E. coli 9218, an N-methyl$N^{\prime}$-nitro- $N$-nitrosoguanidine-derived pep $N$ mutant, was isolated in 1983; however, the nature of the mutation was not identified at that time (Bally et al., 1983). In this report, we wished to determine the nature of the pepN mutation in strain 9218. Towards this goal, we addressed whether PepN was detected in cytosolic extracts in different E. coli strains, by Western blotting using a PepN-specific antiserum. PepN expression was detected at low levels in the wild-type strain E. coli DH5 $\alpha$ and the intensity of PepN increased in extracts from $\mathrm{DH} 5 \alpha$ strains transformed with pBM15, the vector overexpressing PepN (Fig. 2a). Significantly, this band was not detected in extracts of strain 9218; however, PepN was detected in extracts of strain 9218 transformed with pBM15. Together, these results demonstrated that the band recognized by the antiserum was specific to PepN as it was detected in wild-type and in extracts overexpressing PepN but was not detected in pepN mutant strain 9218. To address the molecular nature of the mutation in strain 9218, we PCRamplified and cloned pepN from 9218 under the control of the L-arabinose-inducible promoter $\mathrm{pBAD} 24$, referred to as
pBAD/9218pepN. pBAD/K12pepN or pBAD/9218pepN was transformed into E. coli $\mathrm{DH} 5 \alpha \Delta p e p N$ and induced with $2 \mathrm{mg}$ L-arabinose $\mathrm{ml}^{-1}$ for $4 \mathrm{~h}$. Previously, we demonstrated that PepN is responsible for the hydrolysis of Suc-LLVY-AMC and L-ala-pNA in E. coli (Chandu et al., 2003). Accordingly, these activities were not detected in extracts of E. coli DH5 $\alpha \Delta p e p N$ transformed pBAD24.

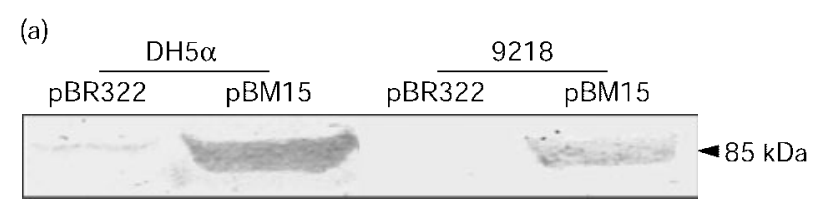

(b)

(b) $\mathrm{DH} 5 \alpha \Delta p e p N$

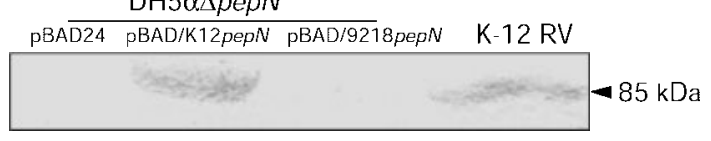

(c)

$\begin{array}{llllllll}\text { K12PepN } & 73 & \text { A } & \text { W } & \text { K } & \text { E } & \text { E } & \text { E } \\ & 217 & \text { gCC } & \text { tgg } & \text { aaa } & \text { gaa } & \text { Gaa } & \text { gag } \\ \text { 9218PepN } & 73 & \text { A } & \text { W } & \text { K } & \mathbf{E} & \text { * } & \\ & 217 & \text { gCC } & \text { tgg } & \text { aaa } & \text { gaa } & \text { Taa } & \text { gag }\end{array}$

Fig. 2. Characterization and mapping of the $p e p N$ mutation in E. coli 9218. (a) Cytosolic extracts of E. coli $\mathrm{DH} 5 \alpha$ and the pepN mutant 9218 transformed with pBR322 or pBM15, the vector overexpressing $\mathrm{PepN}$, were prepared and subjected to Western analysis using the PepN-specific antiserum. (b) pepN was PCR-amplified from E. coli strains K-12 RV and 9218, cloned and expressed in the L-arabinose-inducible pBAD system. Wild-type PepN is represented as pBAD/K12pepN and $9218 \mathrm{PepN}$ is represented as pBAD/9218pepN. The E. coli strain with a targeted deletion in $p e p N$, DH $5 \alpha \Delta p e p N$, was transformed with pBAD24, pBAD/K12pepN or pBAD/ $9218 p e p N$ and the L-arabinose-induced extracts were analysed for PepN expression by Western analysis. (c) Amino acid and nucleotide sequences of pepN from E. coli K-12 RV and E. coli 9218 are represented. 
Overexpression of wild-type K12pepN restored the ability to hydrolyse both Suc-LLVY-AMC and L-ala- $p$ NA. However, overexpression of 9218pepN failed to restore the ability to hydrolyse both these substrates. Transformation and induction of all the three constructs displayed a similar ability to hydrolyse a control peptide substrate, Cbz-LLE- $\beta$ NA (data not shown). These activity data have been further corroborated by Western analysis in Fig. 2(b). As shown in Fig. 2(a), PepN was detected at high levels on overexpression; therefore, we attempted to study the expression of wild-type K-12 and mutant 9218 PepN. PepN expression was not detected in the strain lacking $p e p N$, DH5 $\alpha \Delta p e p N$, transformed with vector alone, pBAD24 (Fig. 2c). However, induction of wild-type PepN (pBAD/K12pepN), but not 9218 PepN (pBAD/9218pepN), resulted in detection of PepN expression in DH5 $\alpha \Delta p e p N$. It is most likely that detection of PepN activity is a more sensitive method compared to detection of PepN by Western analysis (Fig. 2b). Together, activity and expression data suggested that full-length PepN was not synthesized by $9218 p e p N$. Finally, DNA sequence analysis of K12pepN and 9218pepN demonstrated that $9218 p e p N$ differed from the wild-type by a transversion of nucleotide 229 from $G$ to $T$, resulting in Glu-77 of wild-type PepN being replaced by a stop codon (Fig. 2c). Significantly, the putative active site residues of PepN (Gly-261 to Glu-320) are present downstream to this mutation site. Previously, based on agar-based antigenantibody precipitation studies, $\mathrm{PepN}$ in 9218 was thought to be synthesized (Latil et al., 1976). It is not clear whether those results were due to cross-reactivity to truncated PepN in 9218 or non-specific reactivity. By PCR-amplifying pepN from strain 9218 and studying its expression and sequence, we demonstrated that PepN in 9218 is truncated due to a mutation that results in a premature stop codon. This protein is probably unstable and degraded rapidly, resulting in loss in ability to detect protein and enzymic activity (Fig. 2a-c).

\section{Endopeptidase substrate hydrolysis profile of pepN-deficient $E$. coli extracts}

Next, we studied the contribution of PepN to the overall endopeptidase activity in E. coli. The hydrolysis of a panel of endopeptidase substrates was studied in cytosolic extracts of the two pepN mutants, 9218 and DH5 $\alpha \Delta p e p N$, and their respective wild-type strains, K-12 RV and DH5 $\alpha$ (Fig. 3a). The hydrolysis of three endopeptidase substrates, SucLLVY-AMC, Suc-AAF-AMC and Boc-LRR-AMC (partial effect), was reduced (Fig. 3a) in extracts of both pepN mutants. Overexpression of PepN in E. coli 9218 with pBM15 rescued all three activities (Fig. 3b). The rescue of Boc-LRR-AMC was partial as PepN and another uncharacterized serine peptidase (data not shown) were responsible for its hydrolysis. Although we were unable to infer any cleavage preference for endopeptidase substrates by PepN, these results demonstrated that PepN is responsible for hydrolysis of some endopeptidase substrates. As both the mutants were generated in different genetic backgrounds,

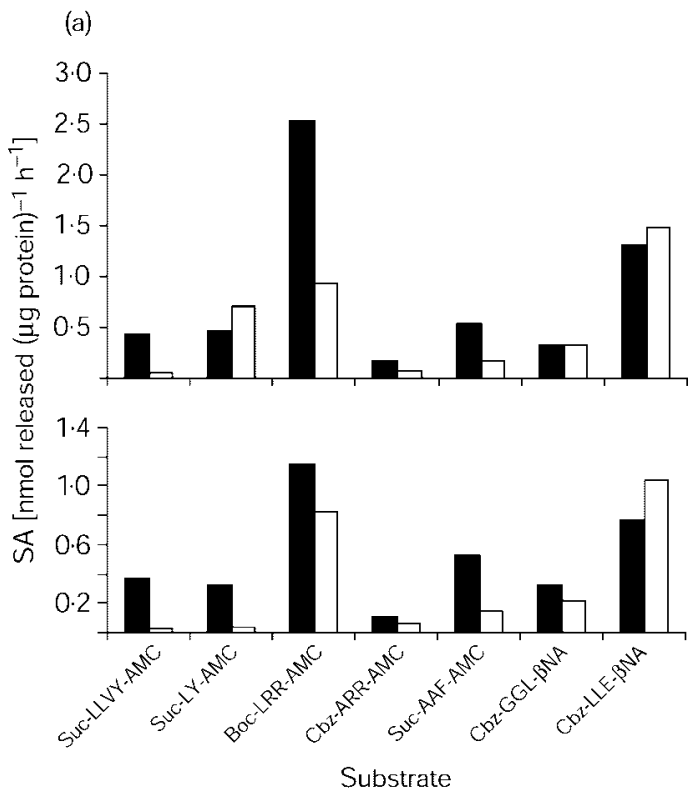

(b)

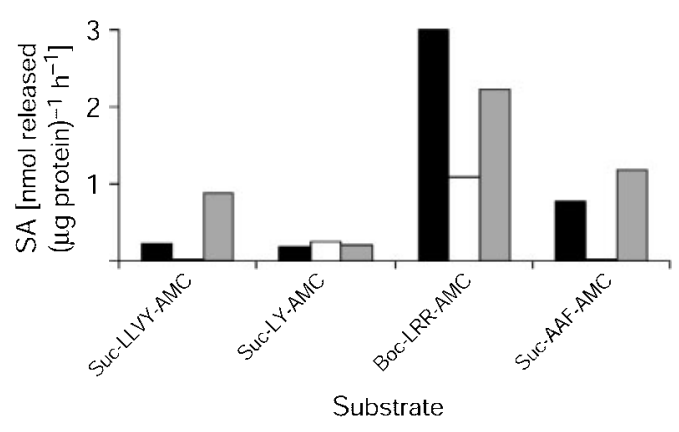

Fig. 3. Hydrolysis profile of endopeptidase substrates. (a) Cytosolic extracts of E. coli strains K-12 RV (top, solid bars), 9218 (top, open bars), DH5 $\alpha$ (bottom, solid bars) and $\mathrm{DH} 5 \alpha \Delta$ pepN (bottom, open bars) were tested for their ability to hydrolyse a panel of endopeptidase substrates. (b) PepN overexpression under its endogenous promoter in $E$. coli 9218 [9218(pBM15), grey bars] compensated the reduced activities in E. coli 9218(pBR322), white bars. E. coli K-12 RV, black bars. SA, specific activity.

effects that were observed in both mutants only were considered as significant.

\section{PepN is the major aminopeptidase in E. coli}

To determine the contribution of PepN to the overall aminopeptidase activities in E. coli, we studied the hydrolysis of a panel of aminopeptidase substrates and an exopeptidase/tripeptidyl peptidase substrate, AAF-AMC, in wild-type and pepN mutant strains $\mathrm{K}-12 \mathrm{RV}$ and 9218 (Fig. 4a, upper) and $\mathrm{DH} 5 \alpha$ and $\mathrm{DH} 5 \alpha \Delta p e p N$ (Fig. 4a, lower). Surprisingly, the hydrolysis of nine out of 13 aminopeptidase substrates was reduced in extracts of $p e p N$ mutants 9218 and $\mathrm{DH} 5 \alpha \Delta p e p N$ compared with the extracts 
(a)

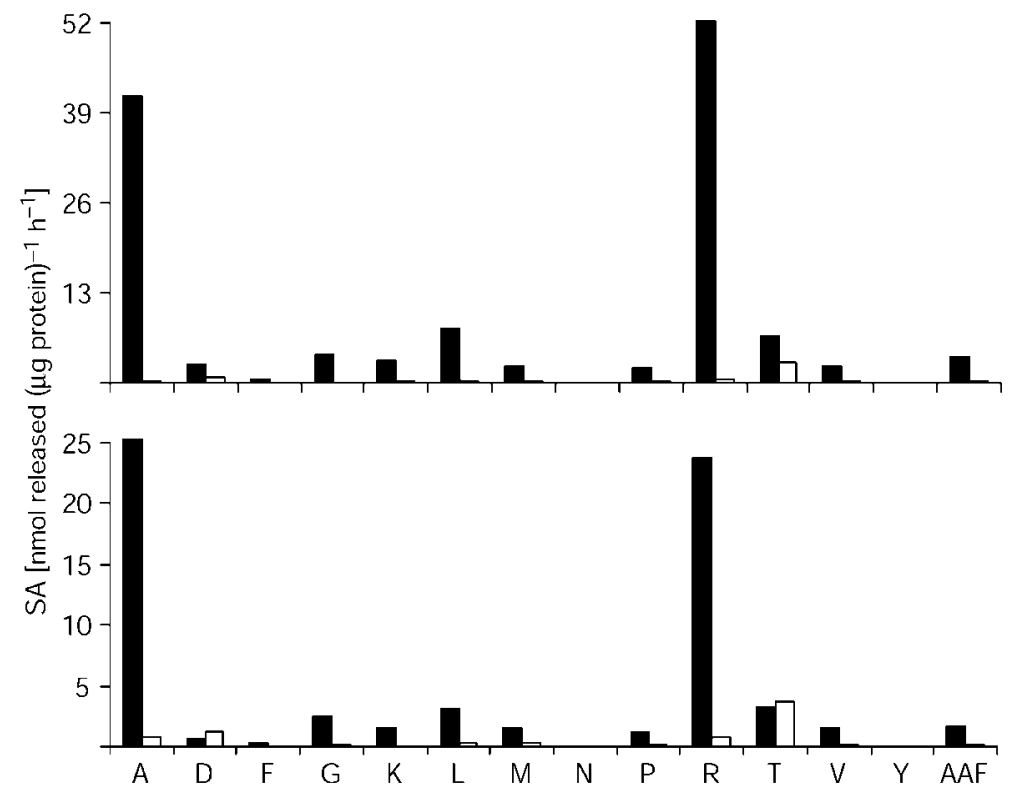

(b)

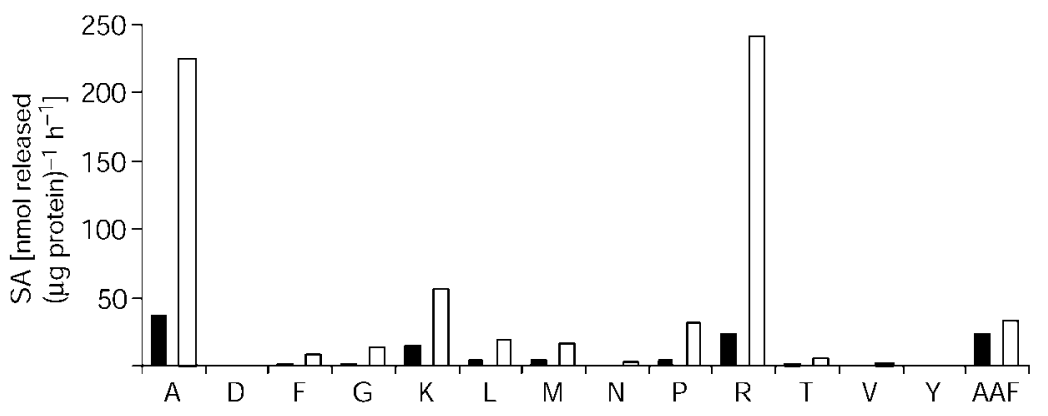

Fig. 4. PepN is responsible for the majority of aminopeptidase activity in $E$. coli extracts. (a) E. coli K-12 RV (top, solid bars) and $\mathrm{DH} 5 \alpha$ (bottom, solid bars) with the respective pepN-deficient strains $E$. coli 9218 (top, open bars) and $\mathrm{DH} 5 \alpha \Delta$ pepN (bottom, open bars) were assayed with a panel of exopeptidase substrates. (b) Extracts of $E$. coli 9218(pBM15), white bars, were tested for ability to compensate the reduced aminopeptidase activities in E. coli 9218(pBR322), grey bars. As expected, extracts from $E$. coli 9218(pBR322) displayed negligible activity. E. coli K-12 RV, black bars. Standard single letter code is used for amino acids in the $\mathrm{P} 1$ position of aminopeptidase substrates. SA, specific activity. of the respective parental strains. Similar loss in hydrolysis was also observed with AAF-AMC, an exopeptidase and tripeptidyl peptidase substrate. PepN overexpression in 9218 by transforming with pBM15 resulted in recovery of all the lost activities (Fig. 4b), suggesting that PepN is responsible for the majority of the aminopeptidase activity in E. coli extracts. Importantly, there was no major loss in hydrolysis of any of the aminopeptidase substrates tested in extracts of peptidase $p e p A$, pep $B$ or $p e p E$ mutant strains compared to the isogenic wild-type strain MG1655 (Fig. 5). Hence, the loss in ability to hydrolyse most of the exopeptidase substrates is specific to $p e p N$.

\section{pepN deficiency confers resistance to NaSal-induced stress}

Previous studies failed to reveal a physiological role for PepN (McCaman et al., 1982; Bally et al., 1983). Recently, genome-wide transcriptional profiling identified induction of the pepN transcript on treatment with $\mathrm{NaSal}$ (Pomposiello et al., 2001) but not heat shock (Richmond et al., 1999). These observations led us to asses the role of PepN during stress using a genetic approach. E. coli strains

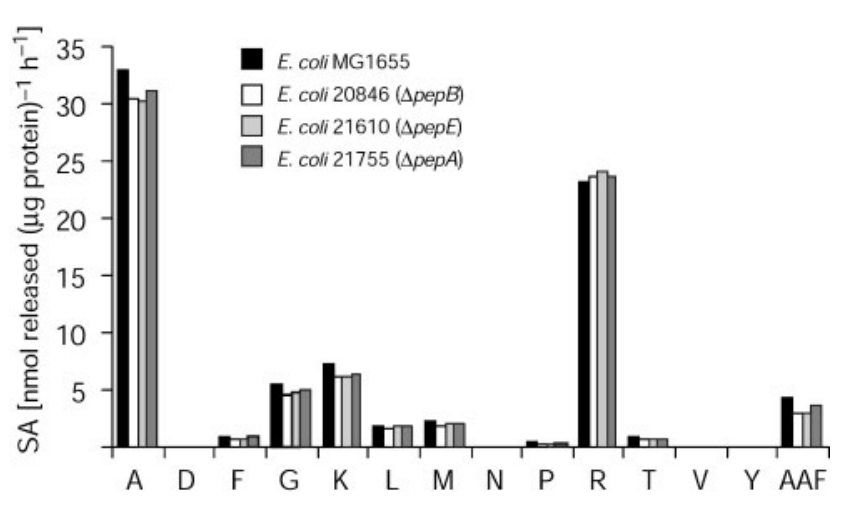

Fig. 5. E. coli PepA, PepB and PepE do not contribute significantly to cleave the exopeptidase substrates tested. Cellular extracts of mutants in $E$. coli peptidases pepA, pepB and pepE were tested for their ability to hydrolyse the panel of exopeptidase substrates. The parent strain E. coli MG1655 was used as a control. Standard single letter code for amino acid residues at $\mathrm{P} 1$ position of aminopeptidase substrates is represented. SA, specific activity. 
DH5 $\alpha$ and DH $5 \alpha \Delta p e p N$ with or without PepN overexpression were subjected to different stress conditions, i.e. growth at high temperature $\left(42^{\circ} \mathrm{C}\right)$ or growth at $37^{\circ} \mathrm{C}$ in the presence of increasing amounts of NaSal. The $\Delta p e p N$ strain grew better than its isogenic wild-type strain in the presence of increasing amounts of NaSal (top two quadrants, Fig. 6a). However, both DH5 $\alpha$ and DH5 $\alpha \Delta p e p N$ strains overexpressing PepN displayed significant loss in ability to grow in the presence of NaSal (bottom two quadrants, Fig. 6a). To test if the observed effect was strain-specific, E. coli 9218 was subjected to growth in the presence NaSal. As observed in Fig. 6(b), E. coli 9218 transformed with pBAD24 displayed significantly better growth compared to 9218/ pBADK12pepN, overexpressing wild-type K-12 PepN (Fig. 6b) from the L-arabinose-inducible promoter (Chandu et al., 2003). Among different stress conditions tested, the role of PepN in NaSal-induced stress was prominent and consistent. Our results using two different strains of E. coli and two different expression systems (one constitutive and the other inducible) demonstrate clearly that PepN acts as a negative modulator during NaSalinduced stress.

(a)

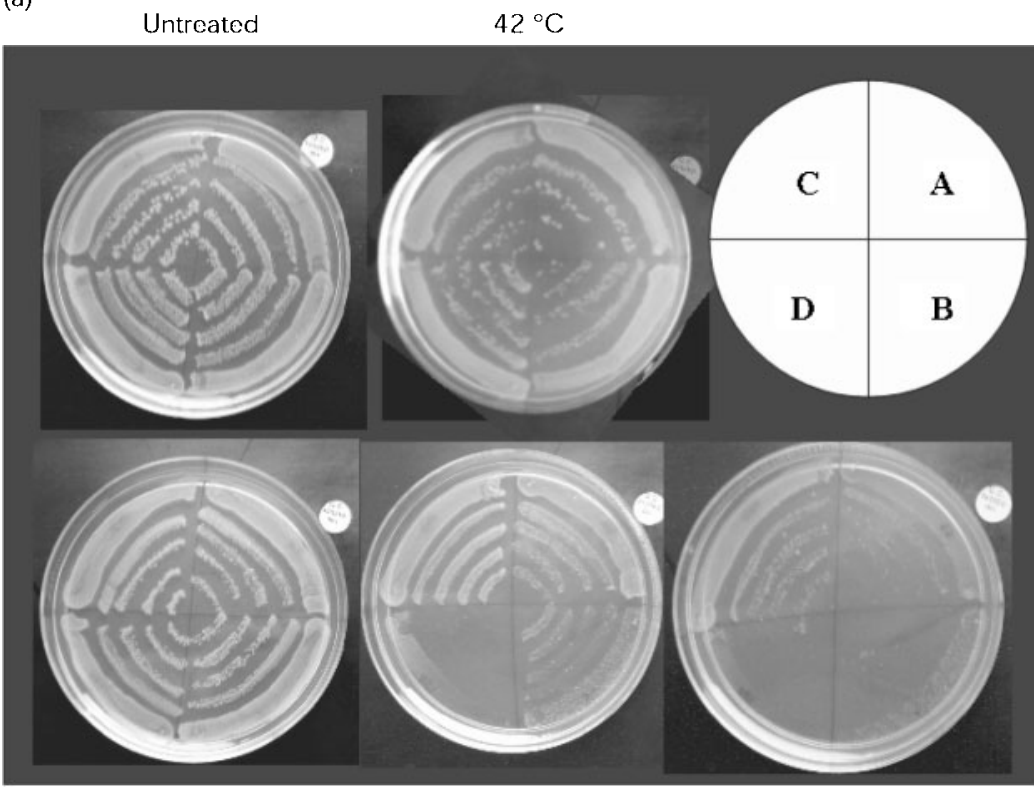

A. E. coli DH5 $\alpha(\mathrm{pBR} 322)$ (wild-type + vector)

B. E. coli DH5 $\alpha(\mathrm{pBM} 15)$ (wild-type + PepN overexpression)

C. E. coli DH5 $\alpha \Delta \rho e p N(\mathrm{pBR} 322)$ (pepN mutant + vector)

D. E. coli DH5 $\alpha \Delta$ pepN(pBM15)

(pepN mutant + PepN overexpression)

$2.5 \mathrm{mM} \mathrm{NaSal}$

$5.0 \mathrm{mM} \mathrm{NaSal}$

$7.5 \mathrm{mM} \mathrm{NaSal}$

(b)

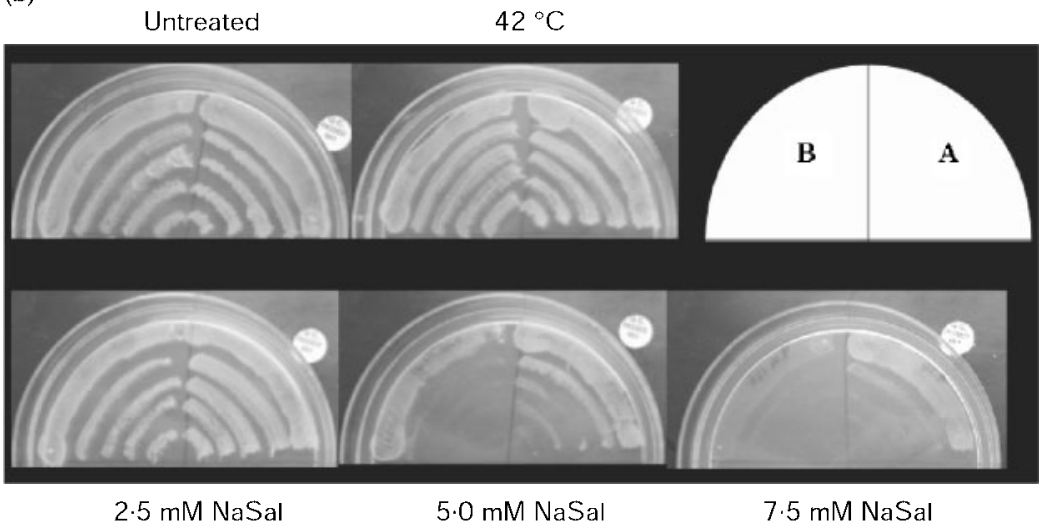

A. E. coli $9218(\mathrm{pBAD} 24)$ (pepN mutant + vector)

B. E. coli $9218(\mathrm{pBAD} 24 / \mathrm{K} 12 \mathrm{pepM})$ (pepN mutant + PepN overexpression)

Fig. 6. PepN expression negatively modulates the response of $E$. coli to NaSal-induced stress. (a) $E$. coli $\mathrm{DH} 5 \alpha$ and $\mathrm{DH} 5 \alpha \Delta$ pepN transformed with vector pBR322 or pBM15, overexpressing PepN, were plated onto LB agar plates supplemented with $30 \mu \mathrm{g}$ tetracycline $\mathrm{ml}^{-1}$ and subjected to growth under different conditions. (b) E. coli 9218 transformed with pBAD24 or pBAD24/K12pepN was grown in the presence of $100 \mu \mathrm{g}$ ampicillin $\mathrm{ml}^{-1}, 400 \mu \mathrm{g} \mathrm{L}$-arabinose $\mathrm{ml}^{-1}$ and varying concentrations of $\mathrm{NaSal}$. The schematic diagrams of the strains in different quadrants are also represented. The results are representative of multiple experiments. 


\section{DISCUSSION}

Using genetic and biochemical approaches, we evaluated the contribution of PepN to the overall peptidase activity in E. coli. Importantly, our studies demonstrate that PepN, but not PepA, PepB or PepE, is responsible for the majority of aminopeptidase activity in E. coli. On the one hand, it is important to point out that the profiles obtained with purified enzyme reflect the ability of the enzyme to hydrolyse a particular substrate. On the other hand, profiles obtained with extracts from wild-type and mutant strains reflect the contribution of the enzyme for overall cellular hydrolysis. Pure PepN cleaved Boc-LRR-AMC as efficiently as SucLLVY-AMC (Fig. 1), whereas results with extracts revealed that PepN is the major Suc-LLVY-AMC-hydrolysing enzyme in E. coli (Chandu et al., 2003) although it contributes partially to the hydrolysis of Boc-LRR-AMC (Fig. 3). Similarly, it is not clear as to the reason for the PepN-specific cleavage of Suc-LY-AMC by strain DH5 $\alpha$ but not by strain 9218 (Fig. 3). However, purified PepN does not cleave Suc-LY-AMC (Fig. 1a). Therefore, comparison of the profiles obtained with both mutant and wild-type extracts, together with data obtained with the pure enzyme, is important in assessing the overall role of PepN in cleaving selected substrates. It is possible that the primary reason for PepN to constitute the majority of aminopeptidase activity in E. coli is the high steady-state levels of this constitutive enzyme. Consequently, the inability to detect differences in cleaving the panel of exopeptidase substrates by PepA, PepB and PepE (Fig. 5) suggests that the steady-state levels of these enzymes are low in E. coli grown in LB medium. Based on the ability to activate an antibiotic, albomycin, a study had predicted that PepA levels are much lower than PepN in E. coli (Braun et al., 1983). Finally, a study has shown that PepN is responsible for $\sim 1 \%$ of total cellular protein in E. coli K-12 (McCaman \& Villarejo, 1982).

Previously, PepN was known to cleave Ala, Lys, Gly, Leu (McCaman \& Villarejo, 1982) and the dipeptide cysteinylglycine (Suzuki et al., 2001). The wide range of substrates used in this study has not been used before and our systematic analysis of the cleavage preference of PepN revealed that it is a broad-specificity aminopeptidase, which cleaves basic and small amino acids substrates better than others (Fig. 1). There are few enzymes that can cleave prolinecontaining peptides; this activity is performed by dedicated enzymes, known as proline iminopeptidases and prolidases (Vanhoof et al., 1995). Interestingly, PepN cleaves L-Pro$\beta \mathrm{NA}$, a proline aminopeptidase substrate. Although PepN is known as the sole alanine aminopeptidase, it cleaves arginine better than alanine. However, the alanine/ arginine aminopeptidase-1 (Aap-1), the PepN homologue in Saccharomyces cerevisiae, cleaves both Arg and Ala (Caprioglio et al., 1993). PepN homologues from L. lactis (van Allen-Boerrigter et al., 1991), Streptococcus thermophilus (Chavagnat et al., 1999) and Aspergillus niger (Basten et al., 2001) are, primarily, lysine aminopeptidases. In general, aminopeptidases involved in protein degradation act on short, not long, peptides (Tamura, N. et al., 1998;
Franzetti et al., 2002). It is possible that PepN preferentially cleaves peptides with basic or small amino acids as aminoterminal residues. Given the specificity of PepN, it is interesting to speculate that, perhaps, other aminopeptidases may be specific for hydrolysis of acidic amino acids, for example, PepB and/or PepE (Larsen et al., 2001). This hypothesis may be justified as the cleavage specificities of aminopeptidases F1, F2 and F3 in T. acidophilum are distinct: F1 is a proline iminopeptidase, F2 prefers basic amino acid substrates and $\mathrm{F} 3$ prefers acidic amino acid substrates (Tamura, $\mathrm{N}$. et al., 1998).

Although there is some information on the role of ATPdependent proteases, for example, Lon and Clp, under stress conditions (Gottesman, 1996; Kuroda et al., 2001), not much is known about the physiological role of ATPindependent peptidases. PepA, PepB, PepD and PepN are thought to be redundant and deficiency in all four peptidases is required to demonstrate an effect on cytosolic protein turnover (Miller \& Schwartz, 1978; Yen et al., 1980). There is a linear relationship between PepN activity and bacterial growth (Bally et al., 1983) and pepN transcription is induced on phosphate starvation, anaerobic conditions and growth in minimal medium (Gharbi et al., 1985). As the significance of these observations is unclear, we resorted to a genetic approach to study the role of PepN under different conditions. The observation that the pepN transcript is induced on NaSal treatment (Pomposiello et al., 2001), but not heat shock (Richmond et al., 1999), prompted us to study the role of PepN under different stress conditions. As shown in Fig. 6, the lack of PepN resulted in increased growth, whereas PepN expression clearly reduced the growth of E. coli, in the presence of NaSal. Thus, PepN is a negative regulator of NaSal-induced stress. The observation that pepN is induced on NaSal treatment and our current result that $\mathrm{PepN}$ is a negative regulator may at first appear to be inconsistent. However, modulation of gene expression, especially microarray data, needs to be confirmed using genetic and/or biochemical approaches (Slonim, 2002). Also, NaSal inactivates the repressor marR and activates marA; however, both these genes are induced on NaSal treatment (Pomposiello et al., 2001). The use of genetic mutants and overexpression studies, as shown in Fig. 6, is the appropriate approach to address the role of pepN under stress. NaSal does not modulate the activity of pure PepN and no difference in PepN activity was found in extracts of untreated or NaSal-treated E. coli (data not shown). Notably, pepN homologues in other organisms are known to play distinct roles. $L$. lactis lacking pepN displayed growth reduction by $20 \%$ in medium containing casein as carbon source (Mierau et al., 1996). Saccharomyces cerevisiae lacking aap-1, a pepN homologue, accumulates less glycogen, whereas, on transforming $\Delta a a p-1$ cells with aap-1, there is more glycogen accumulation (Caprioglio et al., 1993). As glycogen accumulation occurs just as glucose is being exhausted during diauxic growth, glycogen accumulation is considered a marker for stress in yeast. Male mice lacking puromycin-sensitive aminopeptidase, a mammalian PepN 
homologue, are sterile due to impaired spermatogenesis and degenerative morphology of Sertoli cells (Osada et al., 2001a). Female mice lacking puromycin-sensitive aminopeptidase are also sterile due to impaired formation of the corpus luteum (Osada et al., 2001b). PepN and its homologues play distinct roles in the physiology of organisms from different kingdoms. Thus, enzymes involved in downstream processing may play specialized roles in cellular processes.

In mammals, NaSal acts as a non-steroidal anti-inflammatory agent. It is an inducer of heat shock and inhibits the production of inflammatory cytokines, whereas in plants it is part of the host defence system (Price et al., 2000). In prokaryotes, NaSal is used to study the response to a xenobiotic compound. There are several mechanisms by which NaSal acts - dissipation of the proton gradient across the inner membrane, iron chelation, growth inhibition, induction of heat shock and the marA regulon - resulting in the modulation of several genes (Price et al., 2000; Pomposiello et al., 2001). NaSal binds and inactivates MarR and activates MarA, resulting in changes in the outer-membrane profile, decreased permeability to antibiotics and, consequently, increased antibiotic resistance (Cohen et al., 1993; Ramani \& Boakye, 2001). To understand the cellular role of PepN and its relation to certain stress conditions (induced by NaSal, etc.), it is important to consider that it acts as an aminoendopeptidase. In terms of protein turnover, the aminopeptidase function of PepN will be clearly important in the recycling of amino acids. However, we know that there are redundant aminopeptidases which can take over this role (Miller \& Schwartz, 1978; Yen et al., 1980). Therefore, during stress the aminopeptidase activity may be important in cleaving peptides or proteins containing basic or small amino acids which reduce the ability of $E$. coli to display resistance to stress. Arginine and lysine at the amino terminus destabilize proteins and such proteins are targeted for degradation (Gonzales \& RobertBaudouy, 1996). Also, aminopeptidase action may result in isoforms of proteins possessing different amino terminal amino acids (Ishino et al., 1987). Alternatively, PepN via its endopeptidase activity may modulate the proteome of cells. In fact, PepN is required for the activation of the antibiotic albomycin inside E. coli; therefore, pepN mutant cells are resistant to albomycin action. The predicted cleavage sites in albomycin suggest that the endopeptidase activity of PepN plays a role in this cleavage (Braun et al., 1983). It is possible that PepN cleaves cellular proteins that result in decreased ability of E. coli to resist NaSalmediated stress. However, in the absence of PepN, these proteins may be present and help to withstand NaSalinduced stress. Comparison of the proteome of wild-type and pepN mutant strains may identify such cellular substrates of PepN. Further studies are in progress to understand the mechanisms by which the major aminopeptidase in E. coli, PepN, functions as an aminoendopeptidase to negatively modulate the response to NaSal-induced stress.

Thus far, seven aminoendopeptidases have been reported: $\alpha$ - $N$-benzoylarginine- $\beta$-naphthylamide hydrolase (Singh \& Kalnitsky, 1980), hydrolase H (Okitani et al., 1981; Nishimura et al., 1983), PepN (Chandu et al., 2003), bleomycin hydrolase (Koldamova et al., 1998), cathepsin $\mathrm{H}$ (Turk et al., 2001), multicorn (Osmulski \& Gaczynska, 1998) and tripeptidyl peptidase II (Geier et al., 1999). Of these, the last five are involved in cytosolic protein degradation, suggesting an important role for these enzymes in this process. Notably, PepN is the only bacterial aminoendopeptidase characterized (Chandu et al., 2003). PepN is a better aminopeptidase than an endopeptidase, as indeed are other aminoendopeptidases, for example, multicorn (Osmulski \& Gaczynska, 1998) and tripeptidyl peptidase II (Geier et al., 1999). Although purified enzymes involved in downstream processing have been studied in T. acidophilum (Tamura, N. et al., 1998), Schizosaccharomyces pombe (Osmulski \& Gaczynska, 1998) and mouse cells (Geier et al., 1999), there are no studies reported in organisms lacking these enzymes. The extensive substrate preference profile of pure PepN and its role in cellular extracts, together with its physiological role during some stress conditions, are unique to this study and will enhance our understanding of the role of the aminoendopeptidase $\mathrm{PepN}$ in cytosolic protein degradation in E. coli.

\section{ACKNOWLEDGEMENTS}

We thank Dr U. Varshney, Dr D. N. Rao and Dr P. Sadhale for encouraging this study. The microarray paper on modulation of the $p e p N$ transcript during NaSal-induced stress was brought to our attention by Anujith Kumar and we acknowledge his insightful observation. This work was supported by a grant from the Department of Science and Technology, Government of India. D. C. was supported by a fellowship from the Council of Scientific and Industrial Research (CSIR), India.

\section{REFERENCES}

Bally, M., Murgier, M. \& Lazdunski, A. (1983). Molecular cloning and amplification of the gene for aminopeptidase $\mathrm{N}$ of Escherichia coli. FEMS Microbiol Lett 19, 261-265.

Bally, M., Murgier, M. \& Lazdunski, A. (1984). Cloning and orientation of the gene encoding aminopeptidase $\mathrm{N}$ in Escherichia coli. Mol Gen Genet 195, 507-510.

Basten, D. E., Visser, J. \& Schaap, P. J. (2001). Lysine aminopeptidase of Aspergillus niger. Microbiology 147, 2045-2050.

Beninga, J., Rock, K. L. \& Goldberg, A. L. (1998). Interferon- $\gamma$ can stimulate post-proteasomal trimming of the $\mathrm{N}$ terminus of an antigenic peptide by inducing leucine aminopeptidase. J Biol Chem 273, 18734-18742.

Bradshaw, R. A., Brickey, W. W. \& Walker, K. W. (1998). N-terminal processing: the methionine aminopeptidase and $\mathrm{N}$ alpha-acetyl transferase families. Trends Biochem Sci 23, 263-267.

Brandstetter, H., Kim, J. S., Groll, M. \& Huber, R. (2001). Crystal structure of the tricorn protease reveals a protein disassembly line. Nature 414, 466-470.

Braun, V., Gunthner, K., Hantke, K. \& Zimmermann, L. (1983). Intracellular activation of albomycin in Escherichia coli and Salmonella typhimurium. J Bacteriol 156, 308-315. 
Caprioglio, D. R., Padilla, C. \& Werner-Washburne, M. (1993). Isolation and characterization of AAP1. A gene encoding an alanine/ arginine aminopeptidase in yeast. J Biol Chem 268, 14310-14315.

Chandu, D. \& Nandi, D. (2002). From proteins to peptides to amino acids: comparative genomics of enzymes involved in downstream processing events during cytosolic protein degradation. Appl Genom Proteom 1, 235-252.

Chandu, D., Kumar, A. \& Nandi, D. (2003). PepN, the major SucLLVY-AMC-hydrolyzing enzyme in Escherichia coli, displays functional similarity with downstream processing enzymes in archaea and eukarya. Implications in cytosolic protein degradation. J Biol Chem 278, 5548-5556.

Chang, S. Y., McGary, E. C. \& Chang, S. (1989). Methionine aminopeptidase gene of Escherichia coli is essential for cell growth. J Bacteriol 171, 4071-4072.

Chavagnat, F., Casey, M. G. \& Meyer, J. (1999). Purification, characterization, gene cloning, sequencing, and overexpression of aminopeptidase N from Streptococcus thermophilus A. Appl Environ Microbiol 65, 3001-3007.

Cohen, S. P., Levy, S. B., Foulds, J. \& Rosner, J. L. (1993). Salicylate induction of antibiotic resistance in Escherichia coli: activation of the mar operon and a mar-independent pathway. J Bacteriol 175, 7856-7862.

Conlin, C. A. \& Miller, C. G. (1995). Dipeptidyl carboxypeptidase and oligopeptidase A from Escherichia coli and Salmonella typhimurium. Methods Enzymol 248, 567-579.

Coux, O., Tanaka, K. \& Goldberg, A. L. (1996). Structure and functions of the $20 S$ and $26 S$ proteasomes. Annu Rev Biochem 65, 801-847.

Eisenthal, R. \& Cornish-Bowden, A. (1974). The direct linear plot. A new graphical procedure for estimating enzyme kinetic parameters. Biochem J 139, 715-720.

Franzetti, B., Schoehn, G., Hernandez, J. F., Jaquinod, M., Ruigrok, R. W. \& Zaccai, G. (2002). Tetrahedral aminopeptidase: a novel large protease complex from archaea. EMBO J 21, 2132-2138.

Fukasawa, K., Fukasawa, K. M., Kana, M., Fujii, S., Hirose, J. \& Harada, M. (1998). Dipeptidyl peptidase III is a zinc metallo-exopeptidase. Molecular cloning and expression. Biochem J 329, 275-282.

Geier, E., Pfeifer, G., Wilm, M., Luchhiari-Hartz, M., Baumeister, W., Eichmann, K. \& Niedermann, G. (1999). A giant protease with potential to substitute for some functions of the proteasome. Science 283, 978-981.

Gharbi, S., Belaich, A., Murgier, M. \& Lazdunski, A. (1985). Multiple controls exerted on in vivo expression of the pepN gene in Escherichia coli: studies with pepN-lacZ operon and protein fusion strains. J Bacteriol 163, 1191-1195.

Goettig, P., Groll, M., Kim, J. S., Huber, R. \& Brandstetter, H. (2002). Structures of the tricorn-interacting aminopeptidase F1 with different ligands explain its catalytic mechanism. EMBO J 21, 5343-5352.

Gonzales, T. \& Robert-Baudouy, J. (1996). Bacterial aminopeptidases: properties and functions. FEMS Microbiol Rev 18, 319-344.

Gottesman, S. (1996). Proteases and their targets in Escherichia coli. Annu Rev Genet 30, 465-506.

Ishino, Y., Shinagawa, H., Makino, K., Amemura, M. \& Nakata, A. (1987). Nucleotide sequence of the iap gene, responsible for alkaline phosphatase isozyme conversion in Escherichia coli, and identification of the gene product. J Bacteriol 169, 5429-5433.

Kisselev, A. F., Akopian, T. N., Woo, K. M. \& Goldberg, A. L. (1999). The sizes of peptides generated from protein by mammalian $26 \mathrm{~S}$ and $20 \mathrm{~S}$ proteasomes. Implications for understanding the degradative mechanism and antigen presentation. J Biol Chem 274, 3363-3371.

Knipfer, N. \& Shrader, T. E. (1997). Inactivation of the $20 \mathrm{~S}$ proteasome in Mycobacterium smegmatis. Mol Microbiol 25, 375-383.
Koldamova, R. P., Lefterova, I. M., Gadjeva, V. G. \& Lazo, J. S. (1998). Essential binding and functional domains of human bleomycin hydrolase. Biochemistry 37, 2282-2290.

Kuroda, A., Nomura, K., Ohtomo, R., Kata, J., Ikeda, T., Takiguchi, N., Ohtake, H. \& Kornberg, A. (2001). Role of inorganic phosphate in promoting ribosomal protein degradation by the Lon protease in E. coli. Science 293, 705-708.

Larsen, R. A., Knox, T. M. \& Miller, C. G. (2001). Aspartic peptide hydrolases in Salmonella enterica serovar typhimurium. J Bacteriol 183, 3089-3097.

Latil, M., Murgier, M., Lazdunski, A. \& Lazdunski, C. (1976). Isolation and genetic mapping of Escherichia coli aminopeptidase mutants. Mol Gen Genet 148, 43-47.

Lazdunski, A., Murgier, M. \& Lazdunski, C. (1975a). Evidence for an aminoendopeptidase localized near the cell surface of Escherichia coli. Regulation of synthesis by inorganic phosphate. Eur J Biochem 60, 349-355.

Lazdunski, C., Busuttil, J. \& Lazdunski, A. (1975b). Purification and properties of a periplasmic aminoendopeptidase from Escherichia coli. Eur J Biochem 60, 363-369.

Lowe, J., Stock, D., Jap, B., Zwickl, P., Baumeister, W. \& Huber, R. (1995). Crystal structure of the $20 S$ proteasome from the archaeon T. acidophilum at 3. $4 \AA$ resolution. Science 268, 533-539.

Maurizi, M. R. (1987). Degradation in vitro of bacteriophage lambda $\mathrm{N}$ protein by Lon protease from Escherichia coli. J Biol Chem 262, 2696-2703.

McCaman, M. T. \& Villarejo, M. R. (1982). Structural and catalytic properties of peptidase $\mathrm{N}$ from Escherichia coli K-12. Arch Biochem Biophys 213, 384-394.

McCaman, M. T., McPartland, A. \& Villarejo, M. R. (1982). Genetics and regulation of peptidase $\mathrm{N}$ in Escherichia coli K-12. J Bacteriol 152, 848-854.

Mierau, I., Kunji, E. R., Leenhouts, K. J., Hellendoorn, M. A., Haandrikman, A. J., Poolman, B., Konings, W. N., Venema, G. \& Kok, J. (1996). Multiple-peptidase mutants of Lactococcus lactis are severely impaired in their ability to grow in milk. J Bacteriol 178, 2794-2803.

Miller, C. G. \& Schwartz, G. (1978). Peptidase-deficient mutants of Escherichia coli. J Bacteriol 135, 603-611.

Nishimura, T., Okitani, A., Katakai, R. \& Kato, H. (1983). Mode of action towards oligopeptides and proteins of hydrolase $\mathrm{H}$, a highmolecular-weight aminoendopeptidase from rabbit skeletal muscle. Eur J Biochem 137, 23-27.

Okitani, A., Nishimura, T. \& Kato, H. (1981). Characterization of hydrolase $\mathrm{H}$, a new muscle protease possessing aminoendopeptidase activity. Eur J Biochem 115, 269-274.

Osada, T., Watanabe, G., Kondo, S., Toyoda, M., Sakaki, Y. \& Takeuchi, T. (2001a). Male reproductive defects caused by puromycin-sensitive aminopeptidase deficiency in mice. Mol Endocrinol 15, 960-971.

Osada, T., Watanabe, G., Sakaki, Y. \& Takeuchi, T. (2001b). Puromycin-sensitive aminopeptidase is essential for the maternal recognition of pregnancy in mice. Mol Endocrinol 15, 882-893.

Osmulski, P. A. \& Gaczynska, M. (1998). A new large proteolytic complex distinct from the proteasome is present in the cytosol of fission yeast. Curr Biol 8, 1023-1026.

Pomposiello, P. J., Bennik, M. H. \& Demple, B. (2001). Genomewide transcriptional profiling of the Escherichia coli responses to superoxide stress and sodium salicylate. J Bacteriol 183, 3890-3902. Price, C. T. D., Lee, I. R. \& Gustafson, J. E. (2000). The effects of salicylate on bacteria. Int J Biochem Cell Biol 32, 1029-1043. 
Ramani, N. \& Boakye, K. (2001). Salicylate inhibits the translation and transcription of ompF in Escherichia coli. Can J Microbiol 47, 1053-1057.

Richmond, C. S., Glasner, J. D., Mau, R., Jin, H. \& Blattner, F. R. (1999). Genome-wide expression profiling in Escherichia coli $\mathrm{K}-12$. Nucleic Acids Res 27, 3821-3835.

Ruepp, A., Eckerskorn, C., Bogyo, M. \& Baumeister, W. (1998). Proteasome function is dispensable under normal but not heat shock conditions in Thermoplasma acidophilum. FEBS Lett 425, 87-90.

Saric, T., Beninga, J., Graef, C. I., Akopian, T. N., Rock, K. L. \& Goldberg, A. L. (2001). Major histocompatibility complex class I-presented antigenic peptides are degraded in cytosolic extracts primarily by thimet oligopeptidase. J Biol Chem 276, 36474-36481.

Singh, H. \& Kalnitsky, G. (1980). $\alpha-N$-Benzoylarginine- $\beta$-naphthylamide hydrolase, an aminoendopeptidase from rabbit lung. $J$ Biol Chem 255, 369-374.

Slonim, D. K. (2002). From patterns to pathways: gene expression data analysis comes of age. Nat Genet 32 Suppl, 502-508.

Stoltze, L., Schirle, M., Schwarz, G. \& 7 other authors (2000). Two new proteases in the MHC class I processing pathway. Nat Immunol 1, 413-418.

Suzuki, H., Kamatani, S., Kim, E. S. \& Kumagai, H. (2001). Aminopeptidases $\mathrm{A}, \mathrm{B}$, and $\mathrm{N}$ and dipeptidase $\mathrm{D}$ are the four cysteinylglycinases of Escherichia coli K-12. J Bacteriol 183, 1489-1490.

Tamura, N., Lottspeich, F., Baumeister, W. \& Tamura, T. (1998). The role of tricorn protease and its aminopeptidase-interacting factors in cellular protein degradation. Cell 95, 637-648.
Tamura, T., Tamura, N., Cejka, Z., Hegerl, R., Lottspeich, F. \& Baumeister, W. (1996). Tricorn protease - the core of a modular proteolytic system. Science 274, 1385-1388.

Turk, V., Turk, B. \& Turk, D. (2001). Lysosomal cysteine proteases: facts and opportunities. EMBO J 20, 4629-4633.

van Allen-Boerrigter, I. J., Baankreis, R. \& de Vos, W. M. (1991). Characterization and overexpression of the Lactococcus lactis pepN gene and localization of its product, aminopeptidase N. Appl Environ Microbiol 57, 2555-2561.

Vanhoof, G., Goossens, F., De Meester, I., Hendriks, D. \& Scharpe, S. (1995). Proline motifs in peptides and their biological processing. FASEB J 9, 736-744.

Wang, E. W., Kessler, B. M., Borodovsky, A., Cravatt, B. F., Bogyo, M., Ploegh, H. L. \& Glas, R. (2000). Integration of the ubiquitinproteasome pathway with a cytosolic oligopeptidase activity. Proc Natl Acad Sci U S A 97, 9990-9995.

Yen, C., Green, L. \& Miller, C. G. (1980). Degradation of intracellular protein in Salmonella typhimurium peptidase mutants. $J$ Mol Biol 143, 21-33.

York, I. A., Mo, A. X., Lemerise, K., Zeng, W., Shen, Y., Abraham, C. R., Saric, T., Goldberg, A. L. \& Rock, K. L. (2003). The cytosolic endopeptidase, thimet oligopeptidase, destroys antigenic peptides and limits the extent of MHC class I antigen presentation. Immunity 18, 429-440.

Zwickl, P., Baumeister, W. \& Steven, A. (2000). Dis-assembly lines: the proteasome and related ATPase-assisted proteases. Curr Opin Struct Biol 10, 242-250. 